\title{
化学合成的亮氨酸脑啡肽基因 在大肠杆菌中的克隆和表达
}

敖世洲 梁镇和 汤锦炎 周晓雄

丁晓华 陈常庆 李载平

(中国科学院上海生物化学研究所)

\section{摘 要}

化学合成的亮氨酸脑啡肽（LEK）基因与质粒 pBR322 重组, 转化大肠杆菌, 经 过原位杂交篎选，限制性图谱分析和 Southern 杂交鉴定，获得一批 LEK 基因重组 体. 插入乳糖操纵子（lac）启动基因控制 LEK 基因的表达. 一个 lac 转录方向和 LEK 基因转录方向相同的表达质粒，pLE103，能在大肠杆菌中产生 LEK. 用放射 免疫分析方法检测, LEK 的产量可达每毫克细菌蛋白 426 毫微克.

前文报道了亮氨酸脑啡肽（LEK）基因的化学合成 ${ }^{[1]}$ ，本文报告 LEK 基因在大肠杆菌 中的克隆和表达. 苏联 Shemyakin 等人 ${ }^{[2]}$ 曾报道类似工作, 但是我们对重组体的组建采用了 不同的途径. 用乳糖操纵子（lac）的表达调控系统控制 LEK 基因的表达, 使大肠杆菌能够 产生较高水平的亮氨酸脑啡肽.

\section{一、材料和方法}

1. 质粒 DNA 载体 pBR322 由本所徐有成同志提供; 其他重组质粒均按以前报道的方 法 ${ }^{[3]}$ 制备.

2. 酶制剂及其反应条件 限制性内切酶 EcoR1, Bam H1, HindIII 及 T4 DNA 连接酶 由本所冯宗铭、朱绳祖、陆惠芬等同志提供; 酶反应条件见以前工作 ${ }^{[1]}$. 多核苷酸激酶由中国 科学院生物物理所生产; 反应条件见前文 ${ }^{[1]}$. 碱性磷酸酯酶由中国科学院上海细胞生物学所 提供; 反应条件参见 Bano 和 Feix 方法 ${ }^{[5]}$.

3. 细胞转化和平板纱选 用大肠杆菌 C600 或 MC106 作受体菌, 按照 Cohen 等人方法 ${ }^{[6]}$

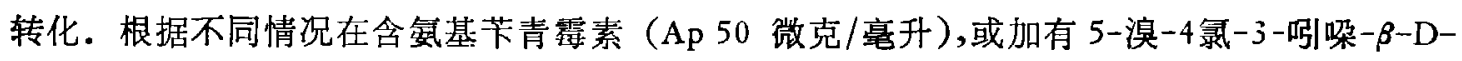
半乳糖苷 (Xgal 40 微克/裹升)的营养琼脂平板上篮选.

4. 分子杂交实验 菌落的原位杂交按 Grunstein 和 Hogness 程序 ${ }^{[7]}$ 处理, DNA 片段的墨 迹转移杂交按 Southern 方法 ${ }^{[8]}$ 进行. 探针为用 $\left[\gamma^{-32} \mathrm{P}\right] \mathrm{ATP}$, 未端标记合成 F2 片段 ${ }^{(32} \mathrm{pTA}$ TGGTGGTTTCCTCTAG $)^{[1]}$. 用 Denhardt 溶液 ${ }^{[9]}$ 杂交, $37^{\circ} \mathrm{C}, 20$ 小时. 用含 $0.5 \% \mathrm{SDS}$ 的 
$2 \mathrm{XSSC} 37^{\circ} \mathrm{C}$ 洗 3 次,每次 1 小时. $\mathrm{X}$ 光片曝光 48 小时.

5. 凝胶电泳条件 $0.7 \%$ 琼脂裙 ${ }^{[1]}$ 和 $20 \%$ 聚丙烯酰胺 ${ }^{[1]}$ 凝胶电泳按以前方法进行.

6. 脑啡肽的放射免疫分析方法 LEK 放射兔疫测定的药箱由上海高血压病研究所供应. 其中包括: ${ }^{125} \mathrm{I}-\mathrm{LEK}$, 非标记的 LEK 标准样品,兔抗 LEK 血清,正常兔血清, 羊抗兔丙种球 蛋白及 PELH 稀释液. 参考陆以信等人方法 ${ }^{[10]}$, 并略加改进.

竞争性抑制标准曲线的实验在 Eppendorf 离心管中进行. 各标准曲线管 (St) 中加人: (1) PELH 缓冲液; (2) 标准品 LEK6.25-400pg/管; (3) ${ }^{125} \mathrm{I}-\mathrm{LEK}$ 约 6,000-8.000 cpm/管; (4) 兔抗 LEK 血清, 使最终工作浓度 $1: 20,000$; 总反应体积为 350 微升. 非特异性结合对照管 (c) 中不加抗血清和标准 LEK. 另制备仅仅加入 ${ }^{125}$ I-LEK 的总计数管 (T). 均做复管. St 及 C 在冰浴中反应 10 小时后加人 1:12 稀释的正常兔血清 15 微升及定量的羊抗兔丙种球蛋白 (20 微升). 继续反应 2.5 小时, $8,000 \mathrm{rpm}$ 离心 5 分钟. 尽可能移去上清液, 沉淀用单道 $r$ 谱 仪测定 cpm. 计算每管的 ${ }^{125} \mathrm{I}-\mathrm{LEK}$ 结合 $(\mathrm{Bi} / \mathrm{T}) \%$, 其中 $\mathrm{Bi}=\mathrm{Sti}-\mathrm{C}$ 以 $(\mathrm{Bi} / \mathrm{T} / \mathrm{Bo} / \mathrm{T})$ 对加人 的 LEK 量或其对数作竞争抑制曲线图.

生长对数期的菌液 5 毫升,离心收集菌体,悬于 1 毫升 $70 \%$ 甲酸含, CNBr 5 毫克,室温黑 暗中反应 24 小时 ${ }^{[1]}$. 加 5 倍体积的水, 冰冻抽干除去甲酸, $\mathrm{pH}$ 近中性, 残留物溶于 2 毫升 PELH 缓冲被内. 样品的测定同前述竞争抑制方法, 以细菌裂解液代替标准品 L.EK. 算出 $(\mathrm{Bi} / \mathrm{T}) \%$ 值，在竞争抑制标准曲线上求得 LEK 的 $\mathrm{pg}$ 数.

7. 细菌蛋白的测定 用于 LEK 放射免疫分析的同样菌液,离心收集菌体，用生理盐水洗 3 次,悬于一定体积的生理盐水中,用 Folin 酚试剂按 Lowry 方法 ${ }^{[2]}$ 测定总蛋白量, 以牛血清 白蛋白作标准曲线。

\section{二、结果和讨论}

图 1 总结了 LEK 基因片段的连接和重组质粒的构建. 前文 ${ }^{[1]}$ 已报道,合成的 LEK 基因 在 $5^{\prime}$ 端带有 EcoR1 粘性末端, 在 $3^{\prime}$ 端有 Bam H1 粘性末端. 质粒 pBR322 经 EcoR1 和 Bam H1 双酶作用, 用 Sepharose-2B 柱分离其中的大片段作为克隆载体. 取 5.pmoles LEK 基 因经多核苷酸激酶作用使 $5^{\prime}$ 端磷酸化, 与 0.5 pmoles 载体在 T4 DNA 连接酶作用下连接之, 构成重组体 $\mathrm{pEK}$, 将此重组体引人大肠杆菌 $\mathrm{C} 600$ 中, 在含 $\mathrm{Ap}$ (50 毫微克/毫升)营养琼脂平 板上获得一批转化体. 按 Grunstejn 和 Hogness 程序 ${ }^{[7]}$ 作菌落原位杂交篮选,同时分离部分转 化体的质粒 DNA，进行 Southern 转移 ${ }^{[8]}$, 对重组体作进一步鉴定. 图 2 为几株 LEK 基因 重组体和载体 $\mathrm{pBR} 322$ 的琼脂糖凝胶电泳及 Southern 转移杂交图. 可以看出除了 $\mathrm{pBR} 322$ 以外,其他 LEK 基因重组体 $\mathrm{pEK} 5,6,7,9$ 和 10 都能与 $5^{\prime}{ }^{-32} \mathrm{p}$ 标记的 LEK 基因 18 核苷 酸片段 (F2 ${ }^{[1]}$ 杂交. pEK5与其他重组体比较, 其电泳迁移速度较慢, 相当于其他重组体的二 聚体.

从所获得的 LEK 基因重组体中, 我们对两个不同类型的重组体 pEK5 和 $\mathrm{pEK10}$ 作进 一步鉴定. 将 pEK5 和 pEK10 DNA 分别经 EcoR1 和 Bam H1 单酶或双酶作用, 作琼脂 糖凝胶电泳和 Southern 转移杂交. 图 3 结果显示, pEK5 (A 和 B) 分别经 EcoR1 (b) 和 Bam H1 (d) 作用后形成线状单体, 在凝胶电泳 (A) 上它们的迁移速度与二聚体的闭环 (CCC) 分子 (a) 相近. 经 $\mathrm{EcoR} 1$ 和 Bam H1 双酶作用 (c), 切去 26 bp LEK 基因, 电泳迁移速度变 


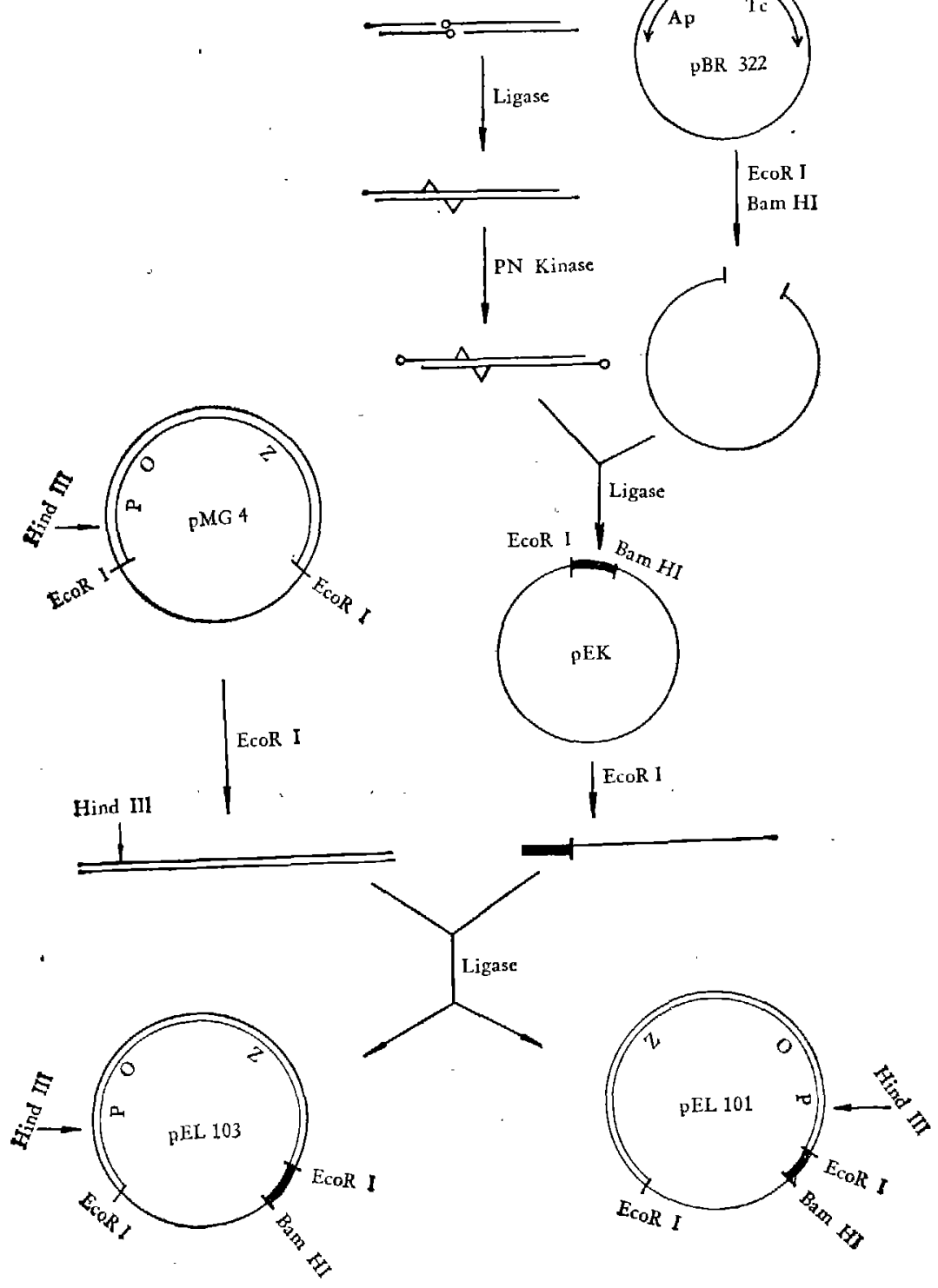

图 1 LEK 基因片段的连接和重组质粒的构建

化不大. 但 Southern 杂交图 (B) 显示, 经 EcoRl 和 Bam H1 双酶作用切去 LEK 基因, 就 不再与 $5^{\prime-32} \mathrm{p}-\mathrm{F} 2$ 探针杂交. $\mathrm{pEK} 10$ ( C 和 D) 经 EcoR1 和 Bam H1 作用也给出同样的结 果,只是被酶作用形成的线形分子较 CCC 分子的电泳迁移速度为德.

在上述琼脂糖凝胶电泳中, 由于 LEK 基因只有 $26 \mathrm{bp}$, 无法观察. 我们又作了 $20 \%$ 聚丙 烯酰胺凝胶 (7M 尿素) 电泳. pEK5 和 $\mathrm{pEK} 10$ 经 EcoR1 和 Bam H1 作用后, 先用磷酸酯酶 处理,再用 $\left[\gamma^{-32} \mathrm{P}\right] \mathrm{ATP}$ 标记5 端. 图 4 为 $20 \%$ 聚丙烯酰胺凝胶电泳放射自显影图. 结果显 示, pEK5 (II) 和 pEK10 (III) 在 EcoR1 和 Bam $\mathrm{Hl}$ 作用下, 都能切出 26 核苷酸的片段, 


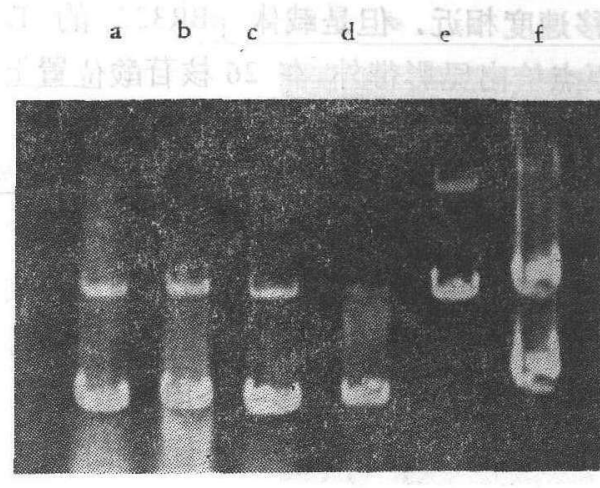

A

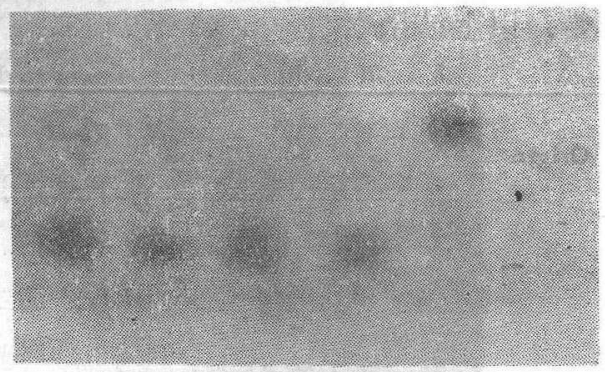

B

图 2 重组质粒的鉴定

A. 重组质煜 $\mathrm{pEK} 5,6,7,9$ 和 10 的琼脂糖凝胶电泳

B. Southern 杂交图. 用 $5^{\prime}{ }^{32} \mathrm{p}-\mathrm{F}_{2}{ }^{32}{ }^{32}$ TATGGTGGTTTCCTCTAG $)^{[1]}$ 作探针.

a. pEk10, b. pEK 9, c. pEK7, d. pEK6 e. pEK 5, f. pRR 322

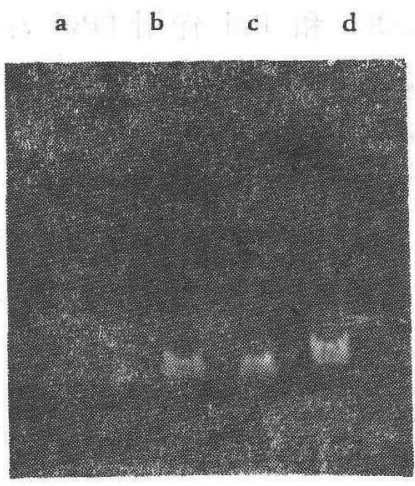

A
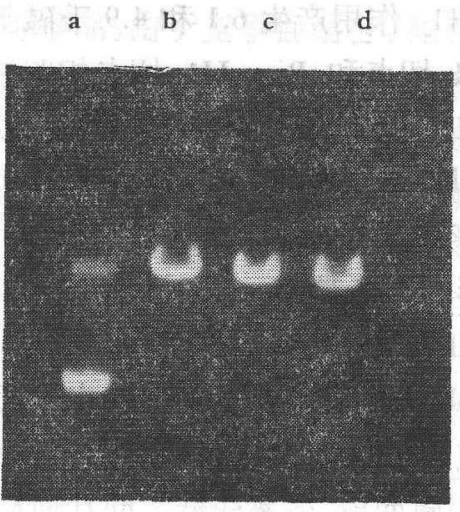

C

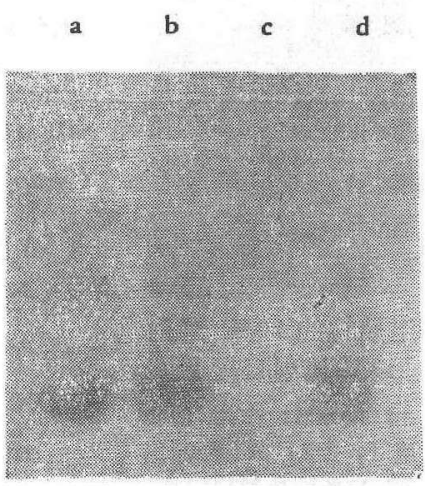

B
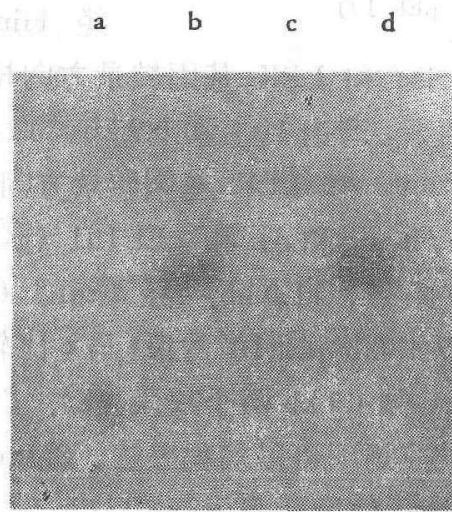

D

图 $3 p \operatorname{EK} 5(\mathrm{~A}, \mathrm{~B})$ 和 $\mathrm{pEK} 10(\mathrm{C}, \mathrm{D})$ 的琼脂糖凝胶电泳图 $(A, C)$ 和 Sonthern 杂交图 (B, D)

(a. ...., b. EcoR1, c. EcoR1 + Bam Hi, d. Bam H1) 
图中可见两条互补链的显影带, 与 XC 标志物迁移速度相近. 但是载体 $\mathrm{pBR} 322$ 的 EcoR1Bam H1 大片段 (I) 经过同样处理, 除在不进胶的原点给出显影带外,在 26 核苷酸位置上没有 显影带. 以上结果表明, pEK5 和 pEK10 均为 LEK 基因的重组体.

\section{II III}

\section{Origin}

$\mathbf{x C}$

BPB

图 4 重组质精的 $20 \%$ 聚丙 烯酰胺凝胶电泳放射自显影图 (经 EcoRI 和 BamH1 作用, 磷酸 酷酶处理, 用 $\left[\gamma-{ }^{32} \mathrm{P}\right]$ ATP 标记 $5^{\prime}$ 端，电泳后作 X光片放射自显影.

I. $\mathrm{pBR} 322$ 对照, II. pEK5, III. pEK 10)

真核基因在原核细胞中表达,都需要原核启动基因控制。 LEK 是一个 5 个氨基酸的小肽,要能在细菌中表达后稳定保 存下来, 还要用细菌的肽段进行保护 ${ }^{[3]}$. 我们用 lac 表达调 控系统控制 LEK 基因的表达，以 $\beta$-半乳糖草酶的肽段保护 LEK. 由于合成的 LEK 基因氨基端多一个甲硫氨酸的密码， 因而在细菌中产生的杂合多肽， $\beta$-半乳糖苗酶肽段和 LEK 之间有一个甲硫氨酸, 经 $\mathrm{CNBr}$ 处理 ${ }^{[1]}$, 甲硫氨酸裂解, 即可 游离出 LEK.

pMG4 为 lac 片段和 pBR322 的重组体 ${ }^{[4]}$, 用 EcoRI 可 以将 lac 片段切出, 然后通过 pEK10 的 EcoR1 切点, 可将 lac 片段插入 pEK10. 如图 1 所示, lac 插入 pEK10 时可能 有 lac 转录方向与 LEK 基因相同和相反的两种方向. 取 0.5 pmoles pMG4 用 EcoR1 和 Pstl 作用 (Pstl 作用使 pBR322 上的 $\mathrm{Ap}^{\mathrm{r}}$ 基因破坏,可减少 $\mathrm{pBR} 322$ 给出的转化体), 切 出 lac 片段; 另取 0.5 pmoles pEK10, 用 EcoR1 切开, 将两 者经 T4 DNA 连接酶作用后转化大肠杆菌 $\mathrm{C} 600$, 在含 Ap (50 微克/毫升) 和 $\mathrm{Xgal}$ (40 微克/㲤升) 营养琼脂平板上饰 选 $\mathrm{Ap}^{\mathrm{r}} \mathrm{lac}^{+}$转化体, 作重组质粒 DNA 分离, 经限制性内切 酶作用,鉴定 lac 的插入方向.

由于 Iac 片段上在启动基因 (P) 的外侧有一个 HindIII 切 点 ${ }^{[14]}$,如果 lac 的转录方向与 LEK 基因转录方向一致,则 lac 上的 HindIII 切点与 LEK 羧端的 Bam Hl 切点相距较远, 经 HindIII 和 Bam H1 作用产生 6.1 和 4.9 干碱基对 ( $\mathrm{Kb}$ ) 的片段; 如 lac 和 LEK 基因转录方向相反, 则 HindIII 切点和 Bam H1 切点相距较近, 在这 两种酶作用下，产生 10.1 和 $0.9 \mathrm{~kb}$ 片段。图 5 为 pEL103 (Iac 和 LEK 基因转录方向相同) 和 pEL101（lac 和 LEK 基因转录方向相反）限制性片段的琼脂糖凝胶电泳图. 可以看出, pEL $103(a, b, c$, 和 $d)$ 和 pEL101 ( $g, h, i$ 和 j) 经 HindIII (a 和 j) Bam H1 (b 和 i) 作用形成一条带, $11.0 \mathrm{~kb}$. 经 EcoR1 (d 和 g) 作用形成两条带: 一条 $7.0 \mathrm{~kb}$ lac 片段（与 $\mathrm{f}$ 比较),一条 4.0kb pEk10 片段 (与 e 比较). 经 HindIII 和 Bam H1 同时作用 ( $\mathrm{c}$ 和 h), pEL103 形成 6.1 和 $4.9 \mathrm{~kb}$ 两个片段 (h). 以上结果表明 pEL103 为 LEK 基因的表达质粒. LEK 基因在 lac 控制下可以实现表达. Shemyakin 等 ${ }^{[2]}$ 报道的表达质粒, 用合成的 LEK 基因, Charon 4A 的 lac 片段和载体 pBR322 三分子反应 1 次组建而成, 效率较低. 而且他们也没有给 出质粒的鉴定结果.

将表运质粒 PEL103 和其他重组质粒分别转人大肠杆菌 C600, 作液体培养, 离心收集 对数生长期的菌体, 经 $\mathrm{CNBr}$ 处理, 作 LEK 放射免疫分析, 测定不同菌体内的 LEK 含量(见 


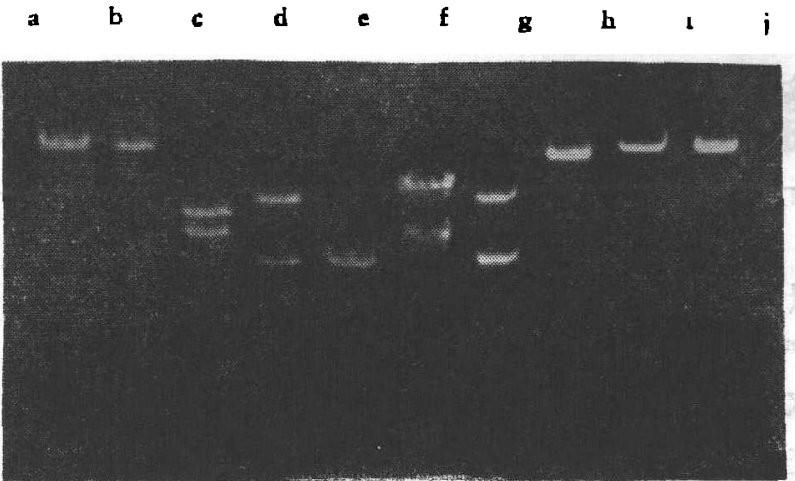

图 5 pEL101 和 pEL103 DNA 限制性片段琼脂糖凝胶电泳图
(a. pEL103 + HindIIl, b. pEL103 + BamH1, c.pEL 103+HindIII + BamHl, d. pEL103 + EcoR1,
e. pEK10 + EcoR1, f.p MG4 + EcoR1, g. pEL101 + EcoR1, h. pEL101 + Hindill + BamH1, i. pEL $101+$ BamHI, j. pEL101 + HindUI)

表 1 亮氮酸脑啡肽的放射免疫分析

\begin{tabular}{|c|c|c|c|}
\hline $\begin{array}{l}\text { 竟 争 物 } \\
\text { (微微克) }\end{array}$ & $\mathrm{cpm}$ & $\begin{array}{l}\text { 细菌 样品 } \\
\text { (蛋白,微克) }\end{array}$ & cpm \\
\hline ............... & 3363 & & \\
\hline $\operatorname{LEK}(6.25)$ & 3026 & C600(10) & 3343 \\
\hline $\operatorname{LEK}(12.5)$ & 2717 & pEK10(18) & 3300 \\
\hline $\operatorname{LEK}(25.0)$ & 2234 & pMG4(20) & 3122 \\
\hline $\operatorname{LEK}(50.0)$ & 1864 & pEL $101(12)$ & 3208 \\
\hline $\operatorname{LEK}(100)$ & 1649 & $\mathrm{pEL103(1.5)}$ & 1212 \\
\hline $\operatorname{LEK}(200)$ & 1270 & pELL103(3.0) & 824 \\
\hline $\operatorname{LEK}(400)$ & 988 & pEL103(7.5) & 740 \\
\hline
\end{tabular}

LEK：亮氨酸-脑・啡肽

材料和方法). 表 1 结果表明, 在测定系统中加入 ${ }^{125} \mathrm{I}-\mathrm{LEK} 6350 \mathrm{cpm}$, 能被双抗免疫沉腚 $3363 \mathrm{cpm}$. 如果在测定系统中另加入非标记 的 LEK 标准品,由于竞争性结合, 能被双抗 免疫沉淀的放射性 (cpm) 减少. 随着非标 记竞争性底物的增加, 被双抗免疫沉淀的放 射性 (cpm) 递减 (表 1 左行). 根据加入非 标记的 LEK 量和被沉淀的放射性 (cpm) 的 改变可以作竞争性抑制标准曲线，以定量未 知样品中的 $\mathrm{LEK}$ 含量. 表 1 右行列出了在 测定系统中加入含不同重组质粒菌体样品时 的被双抗免疫沉淀的放射性 (cpm). 对于大 肠杆菌 C600 以及含有 pEK10，pMG4 和 pEL101 的菌体，在反应系统中加入 $10-20$ 微克的细菌蛋白样品, 被双抗免疫沉淀的放 射性 (cpm) 都没有明显的改变,接近于 3363

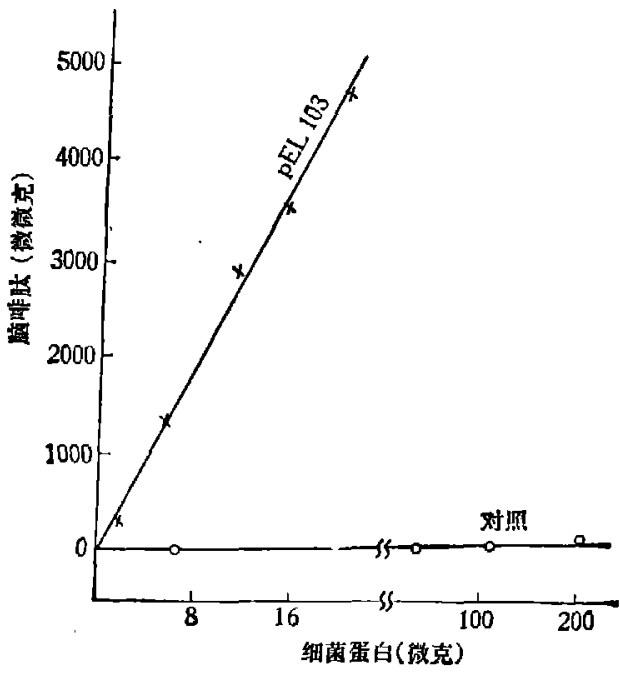

图 6 大肠杆菌 $\mathrm{C} 600$ 产生的亮矢酸脑啡肽 
cpm. 但对含 $\mathrm{pEL} 103$ 表达质粒的菌体,加人细菌蛋白 1.5 微克后，被双抗免疫沉淀的放射性 （cpm）就明显减少,达到 $1212 \mathrm{cpm}$ 。随着细菌蛋白的增加, 放射性（cpm）下降愈明显. 加入 3.0 微克细菌蛋白时,下降到 $824 \mathrm{cpm}$, 加入 7.5 微克细菌蛋白时,降为 $740 \mathrm{cpm}$, 此时 LEK 含量 已超过竞争抑制标准曲线的范围。结果表明含 pEL103 的菌体与其他对照菌比较, 其所产生 的 LEK 是明显的.

$\mathrm{cpm}$ 的改变可以从竞争性抑制标准曲线上换算成 LEK 的量. 从图 6 的结果可以看出, 含 $\mathrm{pEL} 103$ 菌体所产生的 LEK, 随细菌蛋白的增加而升高, 成一直线关系. 在细菌蛋白为 20 微克时,产生 LEK 4,700 微微克. 但 C600 对照菌则不存在这个关系, 细菌蛋白达到 200 微克时, $\mathrm{cpm}$ 的改变只有相当于 18 微微克 LEK 的改变. 这可能是在大量蛋白存在下,对测 定系统的干扰,并不反映 LEK 的产生.

表 2 亮氨酸脑啡肽基因在不同细菌中的表达

\begin{tabular}{c|c|c}
\hline 细 菌 寄主 & ng LEK & $\beta$-gal-LEK 杂合多肽/总蛋白 \\
\hline C600 $\left(\mathrm{i}^{+} \mathrm{z}^{+} \mathbf{y}^{-}\right)$ & 195 & $3.9 \%$ \\
$\operatorname{MC} 106(\Delta \mathrm{lac})$ & 426 & $8.5 \%$ \\
\hline
\end{tabular}

$\beta$-gal: $\beta$-半乳糖葉酸, LEK: 亮氮酸-脑啡肽

大肠杆菌 C600 lac 的表型为 $\mathrm{i}^{+} \mathrm{z}^{+} \mathrm{y}^{-}$, 细胞内有 $\mathrm{i}$ 基因所产生的阻僈蛋白, lac 的表达受 到一定的阻這,利用诱导剂可以提高 lac 的表达水平 ${ }^{[15]}$. 我们改变寄主细胞,将 pEL103 转人 lac 缺失的大肠杆菌 MC 106 ( $(\mathrm{lac}$ )，同样可以使 lac 表达水平提高. 表 2 比较了 pEL103 在 C600 和 MC 106 中的表达水平. 在 C600 中每毫克细菌蛋白产生 LEK $195 \mathrm{ng}, \mathrm{MC} 106$ 则产生 $426 \mathrm{ng}, \mathrm{LEK}$ 的产量增加了一倍以上. 如果换算成 $\beta$-半乳糖苷酶-LEK 杂合蛋白的 产生,在 $\mathrm{C} 600$ 中约占细菌总蛋白的 $3.9 \%$ ，在 MC106 中可达 $8.5 \%$ ，与经异丙基- $\beta-\mathrm{D}-$ 硫代 半乳糖葉 (IPTG) 诱导后的表达水平相近 ${ }^{[2]}$.

本工作承本所冯宗铭、朱绳祖、陆惠苏等同志提供限制性内切酶及 T4 DNA 连接酶; 上 海高血压病研究所邱嘉盛同志对 LEK 放射免疫分析测定提供宝贵意见，谨此致谢。

\section{考文献}

[1] 汤锦炎等, 中国科学,亮一脑啡肽基因的合成(待发表).

[2] Shemyakin, M. F., et al., Nucleic Acids Res., 8(1980), 6163.

[ 3] 教世洲等,迪传学报, 10(1983),85.

[4] 敖世洲、高美华,生物化学与生物物理学报, 14(1982), 121 .

[5] Sano, H. \& Feix, G., Biochemistry, 13 (1974), 5110.

[ 6 ] Cohen, S. N., et al., Proc. Natl. Acad. Sci. USA, 69(1972), 2110.

[ 7 ] Grunstein, M. \& Hogness, D. S., ibid., 73 (1975), 961.

[ 8 ] Southern, E. M., J. Mol. Biol., 98 (1975), 503.

[ 9 ] Denhardt, D. T., Biochem. Biophys. Res. Commun., 23 (1966), 641.

[10」 陆以信等,生物化学与生物物理学报, 12(1980),641。

[11] Gross, E., Methods Enzymol., 11(1967), 238.

[12] Lowry, O. H., et al., J. Biol. Chem., 193 (1951), 265.

[13] Itakura, K., et al., Science, 198 (1977), 1056.

[14] Allent, B. \& Bukhar, A. I., J. Mol. Biol., 92 (1975), 529.

[15] Miller, J. H., Experiments in Molecular Genetics, 1972, 47, Cold Spring Harbor Laboratory, Cold Spring Harbor, New York. 